\title{
Breast Angiosarcoma with a Preoperative Diagnosis of Late Recurrence of Breast Cancer: A Case Report
}

\author{
Rika Kouhashi $^{a}$ Shinichiro Kashiwagi ${ }^{a} \quad$ Yuka Asano $^{a} \quad$ Tamami Morisaki $^{a}$ \\ Sae Ishihara ${ }^{a}$ Wataru Goto ${ }^{a}$ Sayaka Tanaka ${ }^{b}$ Yuko Kuwae $^{b}$ \\ Masahiko Ohsawa $^{b}$ Kosei Hirakawa ${ }^{a}$ Masaichi Ohira ${ }^{a}$ \\ aDepartment of Breast and Endocrine Surgery, Osaka City University Graduate School of \\ Medicine, Osaka, Japan; 'bepartment of Gastrointestinal Surgery, Osaka City University \\ Graduate School of Medicine, Osaka, Japan
}

Keywords

Angiosarcoma $\cdot$ Late recurrence $\cdot$ Breast cancer $\cdot$ Mammary tumor $\cdot$ Surgery

\begin{abstract}
Angiosarcoma is a malignant mesenchymal tumor characterized by the presence of vascular endothelial cells. Although rare, angiosarcoma developing in the mammary glands has a poor prognosis. We report a case of breast angiosarcoma with a preoperative diagnosis of late recurrence of breast cancer. A 78-year-old woman noticed a tumor in her right breast and visited our hospital. The patient had undergone breast-conserving surgery and axillary lymph node dissection from the right breast 12 years before the visit. The tumor was diagnosed as T4bNOM0, stage IIIB. Anastrozole was administered as postoperative adjuvant therapy for 5 years; the patient also received $50-$ Gy whole-breast radiation therapy after surgery. Physical examination during her visit revealed an elevated lesion with blue purpura around the nipple in the right breast. We performed breast ultrasound and detected a well-defined $19.6 \times 16.4 \times$ $10.7 \mathrm{~mm}$ hypoechoic tumor in the left subareolar area. The patient underwent core needle biopsy (CNB). Based on the CNB specimen findings, she was suspected to experience late local recurrence after surgery. Therefore, she underwent total mastectomy after breast-conserving surgery. A dark-red tumor sized $18 \times 12 \mathrm{~mm}$ was found in a specimen from the nipple. The pathological diagnosis of the specimen revealed short spindle-shaped tumor cells with strong nuclear pleomorphism and a significant interstitial fibrosis. Immunohistochemistry using D2-40 and CD31 antibodies showed irregular luminal proliferation at the anastomosis, infiltration into the surrounding tissue, and massive necrosis, thereby leading to the diagnosis of breast angiosarcoma. We have reported a case of breast angiosarcoma with a preoperative diagnosis of late recurrence of breast cancer.




\section{Case Reports in Oncology}

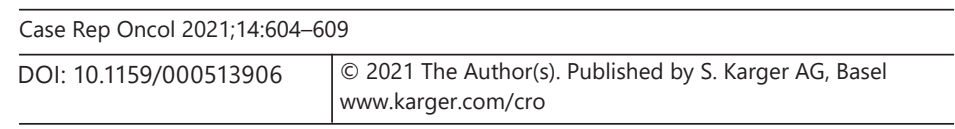

Kouhashi et al.: Breast Angiosarcoma with a Diagnosis of Late Recurrence of Breast Cancer

\section{Background}

Angiosarcoma is a malignant mesenchymal tumor characterized by the presence of vascular endothelial cells. Although rare [1, 2], angiosarcoma developing in the mammary glands has a poor prognosis [3-6]. By using imaging techniques and cytodiagnosis, it has not been possible to characterize angiosarcoma; moreover, tissue biopsy seldom yields an accurate diagnosis of angiosarcoma [7]. We report a case of breast angiosarcoma with a preoperative diagnosis of late recurrence of breast cancer.

\section{Case Presentation}

A 78-year-old woman noticed a tumor in her right breast and visited our hospital. She had undergone breast-conserving surgery and axillary lymph node dissection from the right breast 12 years before the visit. The tumor was diagnosed as T4bN0M0, stage IIIB, invasive ductal carcinoma, estrogen receptor (ER) positive, progesterone receptor (PgR) positive, and human epidermal growth factor receptor (HER) 2 negative; the surgical margin was negative. The nuclear atypia score, mitotic count, and nuclear grade were 3 . Anastrozole was administered as postoperative adjuvant therapy for 5 years; the patient also received 50 -Gy wholebreast radiation therapy after surgery. After completing the adjuvant therapy, she underwent regular follow-up at other hospitals without recurrence. She had no history or family history of addiction.

Physical examination during her visit revealed an elevated lesion with blue purpura around the nipple in the right breast (Fig. 1a). We performed breast ultrasound and detected a well-defined $19.6 \times 16.4 \times 10.7 \mathrm{~mm}$ hypoechoic tumor in the left subareolar area (Fig. 1b, c). Therefore, the patient underwent core needle biopsy (CNB). Following hematoxylin and eosin staining of the CNB specimen, we detected a high-grade necrosis and a high nuclear-cytoplasmic ratio in the tumor cells (Fig. 2a, b). Immunohistochemical analysis revealed the CNB specimen to be cytokeratin (CK) 7, CK20, gross cystic disease fluid protein (GCDFP), ER, PgR and HER2 negative; expression of Ki-67 was high (Fig. 2c). These pathological findings suggested the presence of a poorly differentiated breast tumor. Computed tomography scans did not show any lymph node or distant metastases. Based on these findings, the patient was suspected to experience late local recurrence after surgery. Therefore, she underwent total mastectomy after breast-conserving surgery. A dark-red tumor sized $18.0 \times 12.0 \mathrm{~mm}$ was found in a specimen from the nipple (Fig. 3a). The pathological diagnosis of the specimen revealed short spindle-shaped tumor cells with strong nuclear pleomorphism and a significant interstitial fibrosis (Fig. 3b). Immunohistochemistry was performed, and the tumor cells were found to be vimentin positive and AE1/AE3, CK7, CK20, GCDFP, ER, PgR and HER2 negative; expression of Ki-67 was high. Immunohistochemistry using D2-40 and CD31 antibodies showed irregular luminal proliferation at the anastomosis (Fig. 3c), infiltration into the surrounding tissue, and massive necrosis, thereby leading to the diagnosis of breast angiosarcoma. During the postoperative follow-up period, when no treatment was provided, recurrence was not observed for 6 months after the surgery.

\section{Karger'}



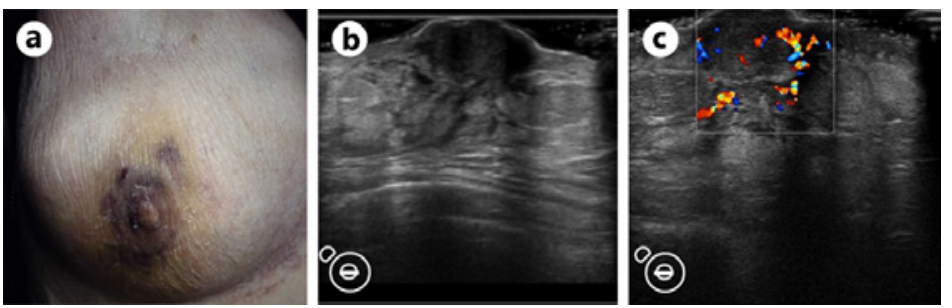

Fig. 1. Physical and ultrasonography findings. a Physical examination during the patient's visit revealed an elevated lesion with blue purpura around the nipple in the right breast. b We performed breast ultrasound and detected a well-defined $19.6 \times 16.4 \times 10.7 \mathrm{~mm}$ hypoechoic tumor in the left subareolar area. c Blood flow rich.
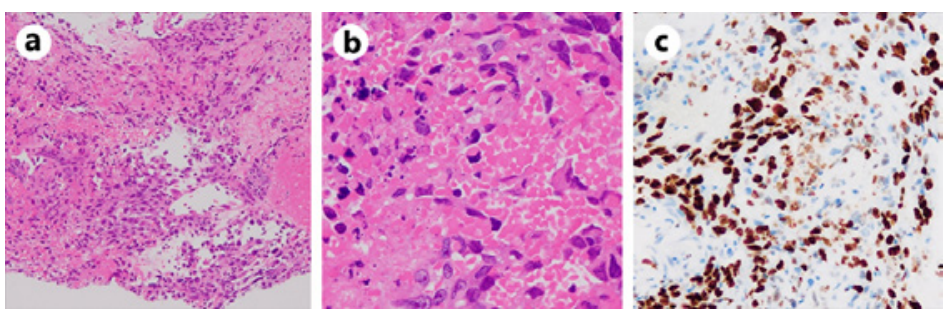

Fig. 2. Pathological findings of the core needle biopsy (CNB) specimen. a, b Following hematoxylin and eosin staining of the CNB specimen, we detected a high-grade necrosis and a high nuclear-cytoplasmic ratio in the tumor cells $(\mathbf{a} \times 100 ; \mathbf{b} \times 400)$. $\mathbf{c}$ Immunohistochemical analysis revealed the CNB specimen to be cytokeratin (CK) 7, CK20, gross cystic disease fluid protein, estrogen receptor, progesterone receptor and human epidermal growth factor receptor 2 negative; expression of Ki-67 was high $(\mathbf{c} \times 200)$.
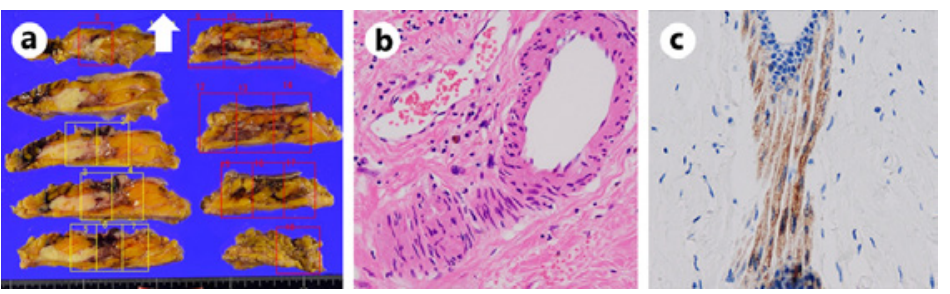

Fig. 3. Macro- and microscopic diagnosis of the resected specimen. a A dark-red tumor sized $18.0 \times 12.0 \mathrm{~mm}$ was found in a specimen from the nipple. $\mathbf{b}$ The pathological diagnosis of the specimen revealed short spindle-shaped tumor cells with strong nuclear pleomorphism and a significant interstitial fibrosis $(\times 200)$. Immunohistochemistry was performed, and the tumor cells were found to be vimentin positive and AE1/AE3, cytokeratin (CK) 7, CK20, gross cystic disease fluid protein, estrogen receptor, progesterone receptor and human epidermal growth factor receptor 2 negative; expression of Ki-67 was high. c Immunohistochemistry using D2-40 (×200) and CD31 antibodies showed irregular luminal proliferation at the anastomosis.

\section{Discussion and Conclusion}

Angiosarcoma, a malignant tumor that originates from the vascular endothelium, is roughly classified into primary and secondary angiosarcoma. Primary angiosarcomas are rare malignancies that form silent tumors in the breast parenchyma. The incidence of primary angiosarcoma is about $0.05 \%$ [1]. On the other hand, secondary angiosarcomas develop in different areas, including the skin, chest wall, and mammary glands after receiving radiation 
therapy for breast cancer. The incidence of secondary angiosarcoma is rising due to the increased use of preservation surgery for the treatment of breast cancer. It has been reported that secondary angiosarcomas usually develop 4-10 years (average, 7 years) after radiation therapy [2]. The patient in our study developed secondary angiosarcoma about 12 years after irradiation following breast-conserving surgery.

Regarding physical findings, the tumor is usually soft, with good mobility, and sometimes has a blue skin or purpura. By using imaging techniques, it has not been possible to characterize angiosarcoma [8, 9]; however, the presence of angiosarcoma has been reported to show a low-intensity signal in T1-weighted magnetic resonance images and a high-intensity signal in T2-weighted images, as well as high-contrast enhancement from the early to the later stages of dynamic study [8,10-12].

Histologically, irregular vascular lumens supported by low- or high-grade tumors proliferate in the mammary tissue and infiltrate into the surrounding adipose tissue $[13,14]$. Because grades vary between patients, it may be difficult to provide a definitive diagnosis from a small biopsy specimen [9]. In other words, in patients with high-grade tumors, it is difficult to differentiate angiosarcoma from carcinoma or other types of sarcoma due to the presence of solid tumors, necrosis, or mitotic figures in tumor cells. In patients with lowgrade tumors, it is difficult to differentiate angiosarcoma from benign hemangioma or pseudoangiomatous stromal hyperplasia. Based on the pathological findings of the CNB specimen, the patient in this study was strongly suspected to have a poorly differentiated breast tumor rather than a high-grade tumor. However, surgical specimens from the patient showed proliferation of high-grade tumor cells with massive necrosis; additionally, after performing immunohistochemistry, the tumor cells were vimentin positive and AE1/AE3 negative. Therefore, the patient was suspected to have malignant mesenchymal tumors. Furthermore, immunohistochemistry for D2-40 and CD31 showed irregular luminal proliferation at the anastomosis, leading to the diagnosis of angiosarcoma.

Surgical resection is the first choice of treatment for breast cancer [15]. Because the possibility of lymph node metastasis is low in patients with angiosarcoma, axillary lymph node dissection may not be required, unless lymph node metastasis is detected [16]. Based on the high incidence of local recurrence and significant reduction in disease-free survival rate in patients with positive surgical margins, surgery achieving a negative surgical margin is desirable $[15,17]$. While some studies reported that immunotherapy (interleukin-2) and chemotherapy (anthracyclines and taxanes) were effective for angiosarcoma treatment [5, 18], no optimal therapeutic strategy has been established yet.

Angiosarcoma has been reported to have a poor prognosis (5-year disease-free survival rate, 44\%; survival rate, 61\%) [3]. Tumor diameter $(>5 \mathrm{~cm})$ and tissue grade (high grade) have been identified as prognostic factors for angiosarcoma [3]. In this study, the patient had a small (tumor diameter, $1.8 \mathrm{~cm}$ ) but high-grade tumor. Therefore, she requires a detailed follow-up in the future to reduce the risk of recurrence.

We reported a case of breast angiosarcoma with a preoperative diagnosis of late recurrence of breast cancer. A deeper understanding of the disease and a comprehensive differential diagnosis are required for effective treatment.

\section{Acknowledgements}

We thank Yayoi Matsukiyo and Tomomi Okawa (Department of Breast and Endocrine Surgery, Osaka City University Graduate School of Medicine) for helpful advice regarding data management.

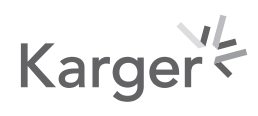




\section{Case Reports in Oncology}

\begin{tabular}{l|l}
\hline Case Rep Oncol 2021;14:604-609 \\
\hline DOI: 10.1159/000513906 & $\begin{array}{l}\text { @ 2021 The Author(s). Published by S. Karger AG, Basel } \\
\text { www.karger.com/cro }\end{array}$ \\
\hline
\end{tabular}

Kouhashi et al.: Breast Angiosarcoma with a Diagnosis of Late Recurrence of Breast Cancer

\section{Statement of Ethics}

Written ethical approval for the publication on the present case report (including images) was obtained from the patient.

\section{Conflict of Interest Statement}

The authors declare that they have no conflicts of interest to disclose.

\section{Funding Sources}

No funding was received for this article and the authors have no conflicts of interest directly relevant to this report.

\section{Author Contributions}

All authors were involved in the preparation of this manuscript. R.K. collected the data and wrote the manuscript. S.K., Y.A., T.M., S.I., and W.G. performed the operation and designed the study. S.K. and K.H. summarized the data and revised the manuscript. S.T., Y.K., and M. Ohsawa performed the pathological diagnosis. K.H. and M. Ohira made substantial contributions to the study design, performed the operation, and revised the manuscript. All authors read and approved the final manuscript.

\section{References}

1 Donnell RM, Rosen PP, Lieberman PH, Kaufman RJ, Kay S, Braun DW Jr, et al. Angiosarcoma and other vascular tumors of the breast. Am J Surg Pathol. 1981 Oct;5(7):629-42.

2 Luini A, Gatti G, Diaz J, Botteri E, Oliveira E, Cecilio Sahium de Almeida R, et al. Angiosarcoma of the breast: the experience of the European Institute of Oncology and a review of the literature. Breast Cancer Res Treat. 2007 Sep;105(1):81-5.

3 Vorburger SA, Xing Y, Hunt KK, Lakin GE, Benjamin RS, Feig BW, et al. Angiosarcoma of the breast. Cancer. 2005 Dec;104(12):2682-8.

4 Zelek L, Llombart-Cussac A, Terrier P, Pivot X, Guinebretiere JM, Le Pechoux C, et al. Prognostic factors in primary breast sarcomas: a series of patients with long-term follow-up. J Clin Oncol. 2003 Jul;21(13):2583-8.

5 Sher T, Hennessy BT, Valero V, Broglio K, Woodward WA, Trent J, et al. Primary angiosarcomas of the breast. Cancer. 2007 Jul;110(1):173-8.

6 Nascimento AF, Raut CP, Fletcher CD. Primary angiosarcoma of the breast: clinicopathologic analysis of 49 cases, suggesting that grade is not prognostic. Am J Surg Pathol. 2008 Dec;32(12):1896-904.

7 Chen KT, Kirkegaard DD, Bocian JJ. Angiosarcoma of the breast. Cancer. 1980 Jul;46(2):368-71.

8 Kikawa Y, Konishi Y, Nakamoto Y, Harada T, Takeo M, Ogata M, et al. Angiosarcoma of the breast - specific findings of MRI. Breast Cancer. 2006 Oct;13(4):369-73.

9 Glazebrook KN, Magut MJ, Reynolds C. Angiosarcoma of the breast. AJR Am J Roentgenol. 2008 Feb;190(2): 533-8.

10 Yang WT, Hennessy BT, Dryden MJ, Valero V, Hunt KK, Krishnamurthy S. Mammary angiosarcomas: imaging findings in 24 patients. Radiology. 2007 Mar;242(3):725-34.

11 Marchant LK, Orel SG, Perez-Jaffe LA, Reynolds C, Schnall MD. Bilateral angiosarcoma of the breast on MR imaging. AJR Am J Roentgenol. 1997 Oct;169(4):1009-10.

12 Murakam S, Nagano H, Okubo K, Sakata H, Tsuji Y, Ishiguro T, et al. Angiosarcoma of the breast: report of a case and its findings of MRI. Breast Cancer. 2001 Aug;8(3):254-8.

13 Voutsadakis IA, Zaman K, Leyvraz S. Breast sarcomas: current and future perspectives. Breast. 2011 Jul;20(3): 199-204.

14 Adem C, Reynolds C, Ingle JN, Nascimento AG. Primary breast sarcoma: clinicopathologic series from the Mayo Clinic and review of the literature. Br J Cancer. 2004 Jul;91(2):237-41. 
15 Kaklamanos IG, Birbas K, Syrigos KN, Vlachodimitropoulos D, Goutas N, Bonatsos G. Breast angiosarcoma that is not related to radiation exposure: a comprehensive review of the literature. Surg Today. 2011 Feb;41(2): 163-8.

16 McGowan TS, Cummings BJ, O’Sullivan B, Catton CN, Miller N, Panzarella T. An analysis of 78 breast sarcoma patients without distant metastases at presentation. Int J Radiat Oncol Biol Phys. 2000 Jan;46(2):383-90.

17 Monroe AT, Feigenberg SJ, Mendenhall NP. Angiosarcoma after breast-conserving therapy. Cancer. 2003 Apr; 97(8):1832-40.

18 Fata F, O'Reilly E, Ilson D, Pfister D, Leffel D, Kelsen DP, et al. Paclitaxel in the treatment of patients with angiosarcoma of the scalp or face. Cancer. 1999 Nov;86(10):2034-7. 